\title{
High virologic response rate after second-line boosted protease inhibitor-based antiretroviral therapy regimens in children from a resource limited setting
}

Thanyawee Puthanakit ${ }^{1,2}$, Gonzague Jourdain ${ }^{3}$, Piyarat Suntarattiwong ${ }^{4}$, Kulkanya Chokephaibulkit ${ }^{5}$, Umaporn Siangphoe ${ }^{1}$, Tulathip Suwanlerk', Wasana Prasitsuebsai ${ }^{1,5}$, Virat Sirisanthana ${ }^{6}$, Pope Kosalaraksa ${ }^{7}$, Witaya Petdachai ${ }^{8}$, Rawiwan Hansudewechakul ${ }^{9}$, Naris Waranawat ${ }^{4}$ and Jintanat Ananworanich ${ }^{1,10,11^{*}}$ On behalf of the HIV-NAT 086 study team

\footnotetext{
Abstract

Background: Limited data exist for the efficacy of second-line antiretroviral therapy among children in resource limited settings. We assessed the virologic response to protease inhibitor-based ART after failing first-line nonnucleoside reverse transcriptase inhibitor (NNRTI)-based regimens.

Methods: A retrospective chart review was conducted at 8 Thai sites of children who switched to PI -based regimens due to failure of NNRTI -based regimens. Primary endpoints were HIV RNA $<400$ copies/ml and CD4 change over 48 weeks.
}

Results: Data from 241 children with median baseline values before starting Pl-based regimens of 9.1 years for age, $10 \%$ for CD4\%, and $4.8 \log _{10}$ copies/ml for HIV RNA were included; 104 (41\%) received a single ritonavir-boosted PI (sbPI) with 2 NRTIs and 137 (59\%) received double-boosted PI (dbPI) with/without NRTIs based on physician discretion. SbPI children had higher baseline CD4 (17\% vs. 6\%, p<0.001), lower HIV RNA (4.5 vs. 4.9 log 10 copies/ml, $\mathrm{p}<0.001)$, and less frequent high grade multi-NRTI resistance $(12.4 \% \mathrm{vs} 60.5 \%, \mathrm{p}<0.001)$ than the $\mathrm{dbPI}$ children. At week $48,81 \%$ had HIV RNA < 400 copies/ml (sbPI 83.1\% vs. dbPI 79.8\%, p=0.61) with a median CD4 rise of 9\% (+7\%vs. + 10\%, p < 0.005). However, only $63 \%$ had HIV RNA $<50$ copies $/ \mathrm{ml}$, with better viral suppression seen in sbPI (76.6\% vs. $51.4 \%$, p 0.002$)$.

Conclusion: Second-line PI therapy was effective for children failing first line NNRTI in a resource-limited setting. $\mathrm{DbPI}$ were used in patients with extensive drug resistance due to limited treatment options. Better access to antiretroviral drugs is needed.

Keywords: Pediatric HIV, Drug resistance, Second-line antiretroviral therapy, Protease-inhibitors, Resource limited settings

\footnotetext{
* Correspondence: Jintanat.A@hivnat.org

${ }^{1}$ The HIV Netherlands Australia Thailand Research Collaboration (HIV-NAT), Bangkok, Thailand

${ }^{10}$ South East Asia Research Collaboration with Hawaii, Hawaii, Thailand

Full list of author information is available at the end of the article
} 


\section{Introduction}

The most commonly used first-line antiretroviral therapy (ART) in HIV-infected children in resource-limited settings (RLS) is a non nucleoside reverse transcriptase inhibitor (NNRTI)-based treatment[1,2]. Data from individual cohorts in Thailand [3], Uganda [4] Cambodia [5] and those from a meta-analysis of 1457 children [1] showed that $70-81 \%$ had viral suppression after 1 year of first-line treatment. Several pediatric programs in RLS have begun to provide second-line therapy; $5.8 \%$ and $20 \%$ among cohorts of HIV-infected children in South Africa [6] and the TREAT Asia regional network [7], but treatment outcomes data are limited. The second-line regimen for children failing NNRTI-based treatment in all treatment guidelines is a low-dose ritonavir boosting protease inhibitors (boosted PI) in combination with 2 nucleoside reverse transcriptase inhibitors (NRTIs) $[8,9]$ which was reasonably effective [10].

However, in RLS without routine virologic monitoring, the diagnosis of treatment failure is usually late, of which brings about concerns for multi-NRTI resistance [11]. Reported incidence rate of multi-NRTI drug resistance from cohort with routine viral load monitoring is only $8 \%$ [12] compared to $36 \%$ seen in settings in which treatment failure was detected by clinical or immunologic criteria [13]. In such cases in RLS, the only effective anti-retroviral (ARV) drug option available for secondline therapy is boosted PI with limited choices for effective NRTIs. Often times, double-boosted PI regimens are used to provide 2 active agents in the regimen. Doubleboosted PIs is an alternative second-line therapy option in the Thai National Guideline; however, there have been limited data of its safety and efficacy in HIV-infected children [14].

The primary objective of this study is to describe the immunologic and virologic efficacy of second-line ARV regimens containing either single- or double-boosted PI over a 48-week period in children with NNRTI-based treatment failure in Thailand.

\section{Material and methods}

\section{Study design and subjects}

We formed a network of 8 large pediatric HIV centers in Thailand to retrospectively collect treatment outcome data of all children who failed NNRTI-based therapy and received ritonavir-boosted PI regimen as a second-line drug regimen. Immunologic treatment failure was defined according to the Thai guideline either CD4 percentage decline $>5$ percentage point in a patient with CD $4 \%$ less than 15 , or CD 4 cell count drop $>30 \%$ of baseline within 6 months [15]. Before 2006, the access to plasma HIV RNA monitoring was limited and it was performed after immunologic failure was suspected. Virological failure was defined as HIV RNA $>1000$ copies/ml after at least 6 months of antiretroviral therapy. After 2006, annual HIV RNA was accessible through the national program. In some cases, genotypic resistance testing was performed before switching to second-line regimens, and the test was performed only if plasma HIV RNA was $>1,000$ copies/ml.

Cases selected were HIV-infected children aged $<18$ years, with a documented history of immunologic or virologic failure on NNRTI-based ART who received ritonavir-boosted PIs-based regimen for at least 24 weeks. They were excluded if they had previously received PIs treatment prior to the ritonavir-boosted PIs for longer than 30 days, or received second-line drugs not belonging to the NRTI, NNRTI, and PI classes. Standardized forms were used for retrospective hospital chart extraction: include demographics, CDC HIV clinical classification, history of ART, CD4 cell count and percentage, and genotypic resistance test result before switching to PI-based HAART. Follow up CD4, plasma HIV RNA and adverse events after switch to PI regimen were extracted and censored at last patient visit. After switching to second-line therapy, CD4 was uniformly monitored every 6 months and HIV RNA every 612 months. Single-boosted PI was defined as low dose ritonavir combined with one other PI drug. Doubleboosted PI was defined as low dose ritonavir combined with another two PI drugs, or therapeutic dose ritonavir (350-400 $\mathrm{mg} / \mathrm{m}^{2} /$ dose) combined with one other PI drug.

The HIV RNA testing was performed using 50 copies/ $\mathrm{ml}$ as the limit of detection, except for some cases in the early 2000s which used 400 copies/ml as the detection limit. Genotypic resistance testing was performed by TrueGene HIV-1 Genotyping system (Visible Genetics, Inc., Toronto, Canada) at 5 sites, ViroSeq HIV-1 Genotyping System (Celera Diagnostics, Alameda, Calif.) at 1 site and with an in house method using Stanford and International AIDS society (IAS) database [16] at 2 sites. The study was approved by the Ethics Committees at all sites.

\section{Statistical analysis}

Primary endpoints were the proportion of children with plasma HIV RNA $<400$ copies $/ \mathrm{ml}$ at week 48 and the CD4 changes at 48 weeks after switching to second-line PI therapy. Secondary endpoints were the proportion of children with plasma HIV RNA $<50$ copies/ml and the prevalence of adverse events. The analysis was performed using available data.

The cumulative probability of virologic treatment failure defined as HIV RNA $>400$ copies $/ \mathrm{ml}$ after at least 6 months of second-line boosted PI regimen was calculated using the Kaplan Meier estimates. The data included patients who had HIV RNA result at least once 
after 24 weeks of treatment and censored at 24 months or earlier in cases of discontinuation of the follow-up. The difference between single- and double -boosted PI was tested using the log rank test.

The predictors for treatment failure defined as HIV RNA $<400$ copies $/ \mathrm{ml}$ at week 48 were explored in a logistic regression model. Factors included CD4 cell count at time of switch to boosted PI regimen (dichotomized as $>100$ cells $/ \mathrm{mm}^{3}$ ), plasma HIV RNA at time of switch to boosted PI regimen (dichotomized as $>10,000$ copies/ $\mathrm{ml}$ ), ART regimen as single-boosted PI or doubleboosted PI, use of lopinavir/ritonavir (LPV/r) -containing regimen, and grade of resistance mutations. Definition of high grade multi-NRTI resistance was defined as $\geq 4$ thymidine analog mutations (TAMs) or the presence of Q151M or 69 insertion. Low grade multi-NRTI resistance was defined as 1 to 3 TAMs. Factors with pvalue $<0.2$ in the univariate analysis were tested in the multivariate analysis. Analyses were performed using SAS version 9.1(SAS Institute, Cary, NC, USA).

\section{Results}

\section{Patient characteristics and antiretroviral regimen}

Between September 2002 and June 2007, 241 children were enrolled, including $1,11,62,63,60$, and 44 children per calendar year. There were 104 children who switched to single-boosted PI and 137 children who switched to double-boosted PI (137 children) at $8 \mathrm{HIV}$ pediatric clinics. The baseline characteristics of the children are shown in Table 1. The medians (interquartile range [IQR]) were 9.1 (7.2-11.1) years for age and 2.2 (1.5-2.9) years for duration of NNRTI-based regimen. Among 203 children who had genotypic resistance testing performed, 195 children (96\%) had the test result prior to switching to PI-based regimen and the remaining had the test done on stored samples at a later date. The ARV regimens were chosen by physician's discretion based on ARV treatment history, genotypic resistance pattern (if available), and the availability of ARV drugs. Indinavir has been available in national program, since 2002, while LPV/r has been available in national antiretroviral program since 2005. The median follow-up time was 26.0 months (IQR 12.7-36.8). Overall, at the time of switch to second line regimens, the children who were prescribed single-boosted PI were younger (median: 8.9 versus 9.4 years, $\mathrm{P}=0.046$ ), had a significantly higher baseline CD4\% (17\% versus 6\%, p<0.001), a lower baseline plasma HIV RNA (4.5 $\log _{10}$ versus 4.9 $\log _{10}$ copies/ml; p <0.001), and a lower proportion of genotypes with multi-NRTI resistance mutation $(12.4 \%$ versus $60.5 \%, \mathrm{p}<0.001)$ compared with children who received double-boosted PI regimen.

The single-boosted PI regimens included 55 (53\%) indinavir/r (IDV/r) and 49 (47\%) lopinavir/r (LPV/r).
The NRTI backbones used were 50 (48\%) zidovudine (AZT) plus lamivudine (3TC), 29 (28\%) AZT plus didanosine (ddI), 17 (16\%) ddI plus 3TC and 8 (8\%) miscellaneous. Among children in the double -boosted PI group, 77 (56\%) did not receive any NRTI, 37 (27\%) received $3 \mathrm{TC}$ as the only NRTI, and $23(17 \%)$ received other NRTIs. The double-boosted PI regimens were $\mathrm{LPV} / \mathrm{r}+\mathrm{IDV}$ in $46(34 \%)$ children, IDV $/ \mathrm{r}+$ Nelfinavir (NFV) in 24 (18\%), $\mathrm{LPV} / \mathrm{r}+$ Saquinavir (SQV) in 20 (15\%), $\mathrm{LPV} / \mathrm{r}+\mathrm{NFV}$ in $17(12 \%), \mathrm{IDV}+\mathrm{RTV}$ in $16(12 \%)$, $\mathrm{IDV} / \mathrm{r}+\mathrm{SQV}$ in $8(6 \%)$ and other PIs in $6(4 \%)$ children.

\section{Efficacy of second-line boosted protease inhibitor regimens}

At week 24, 223 had CD4 information and 190 had plasma HIV RNA information. The overall median (IQR) CD4\% change was $5(1-8) \%$ with more CD4 rise in children who received double- compared to singleboosted PI $(+5 \%$ vs. $+4 \%, \quad \mathrm{p}<0.016)$. Overall $78 \%$ achieved HIV RNA $<400$ copies $/ \mathrm{ml}$. The proportion with HIV RNA $<400$ copies/ml was not different between groups; $80.9 \%$ (72/89) in single- vs. $76.2 \%$ (77/ $101)$ in double-boosted PI regimens. The proportion with HIV RNA below 50 copies/ml was $59 \%$ overall with a higher proportion in single- compared to double-boosted PI groups: $66.7 \%(58 / 87)$ vs. $51.2 \%(44 / 86) \mathrm{p}=0.038)$.

The outcomes at week 48 after initiation of boosted PI regimens are shown in Table 2. The increase in CD4 percentage was significantly higher among children who received double-boosted PI. Overall, 81.3\% had HIV RNA $<400$ copies/ml (single-versus double-boosted PI: $83.1 \%$ vs. $79.8 \%, p=0.61)$. Sixty-three percent had plasma HIV RNA < 50 copies/ml; a higher proportion of these were children who received single-boosted PI compared with those receiving double-boosted PI (76.6\% vs. $51.4 \%, \mathrm{p}=0.002$ ).

Subgroup analysis was performed for the outcome HIV RNA $<400$ copies/ml at week 48. Among children in single-boosted PI group, 30/36 (83.3\%) of children who received indinavir/ $\mathrm{r}$ and $24 / 29(82.8 \%)$ of children who received lopinavir/ $\mathrm{r}$ had undetectable viral load (Pvalue 0.655). Among children in double boosted PI group, $47 / 57$ (82.5\%) of children who did not receive any NRTI and 16/22 (72.7\%) of children who received NRTIs had undetectable viral load (p-value 0.252).

\section{Adverse events}

During the follow-up period, there were 31 adverse events that led to discontinuation or substitution of PI drugs. The median time of discontinuation or substitution were 13.3 months (IQR 7.1-26.0) after start PIbased regimen. There were 20 events for IDV-related toxicity (13\% of children who used IDV)due to nephrotoxicity $(\mathrm{n}=11)$, hyperbilirubinemia $(\mathrm{n}=4)$, nausea 
Table 1 Baseline Characteristics among 241 HIV-infected children who received second-line protease inhibitor -based antiretroviral therapy

\begin{tabular}{lllll}
\hline Characteristics & $\begin{array}{l}\text { All } \\
(\mathbf{n}=\mathbf{2 4 1 )}\end{array}$ & $\begin{array}{l}\text { Single-boosted PIRegimens } \\
(\mathbf{n}=\mathbf{1 0 4})\end{array}$ & $\begin{array}{l}\text { Double-boosted PI regimens }^{\mathbf{1}} \\
(\mathbf{n}=\mathbf{1 3 7})\end{array}$ \\
\hline Gender: Male & $117(49)$ & $55(53)$ & $62(45)$ & 0.24 \\
Age, years & $9.1(7.2-11.1)$ & $8.9(6.1-11.1)$ & $9.4(7.6-11.2)$
\end{tabular}

Prior to initiate NNRTI regimen

CDC clinical staging

$\begin{array}{lll}\text { N } & 10(4.2) & 7(6.7) \\ \text { A } & 58(24.3) & 29(27.9) \\ \text { B } & 113(47.3) & 39(37.5) \\ \text { C } & 58(24.3) & 29(27.9) \\ \text { CD4 percentage } & 5(1-10) & 3.5(1-11) \\ \text { CD4 cell/mm } \text { m }^{3} & 109(27-410) & 109(21-395)\end{array}$

$3(2.2)$

29 (21.6)

$74(54.8)$

$29(21.5)$

$5(2-10)$

0.53

$112(28-413)$

0.51

Prior to switch to second-line boosted -PI regimen

\begin{tabular}{|c|c|c|c|c|}
\hline Weight for age Z-score & $-1.7(-2.1$ to -0.9$)$ & $-1.4(-1.9$ to -0.7$)$ & $-1.9(-2.3$ to -1.4$)$ & $<0.001$ \\
\hline CD4\% $(n=239)$ & $10(4-18)$ & $17(7-24)$ & $6(2-12)$ & $<0.001$ \\
\hline CD4 cell $/ \mathrm{mm}^{3}(\mathrm{n}=238)$ & $195(70-442)$ & $379(165-659)$ & $123(38-273)$ & $<0.001$ \\
\hline HIV RNA, log 10 copies/mL $(n=227)$ & $4.8(4.3-5.3)$ & $4.5(3.9-5.1)$ & $4.9(4.5-5.4)$ & $<0.001$ \\
\hline \multicolumn{5}{|l|}{ Antiretroviral treatment history } \\
\hline $\begin{array}{l}\text { Mono/dual NRTI exposure prior } \\
\text { to NNRTI-based regimen }\end{array}$ & $71(29.5)$ & $23(22.1)$ & $48(35.0)$ & 0.03 \\
\hline Duration on NNRTI-based treatment, years & $2.2(1.5-2.9)$ & $2.3(1.5-3.1)$ & $2.0(1.4-2.9)$ & 0.04 \\
\hline NNRTI regimen & & & & 0.01 \\
\hline Nevirapine & $167(69.3)$ & $81(77.9)$ & $86(62.8)$ & \\
\hline Efavirenz & $74(30.7)$ & $23(22.1)$ & $51(37.2)$ & \\
\hline \multicolumn{5}{|l|}{ NRTI-backbone } \\
\hline $\mathrm{d} 4 \mathrm{~T} / 3 \mathrm{TC}$ & 142(58.9) & $71(68.2)$ & $71(51.8)$ & 0.015 \\
\hline AZT/3TC & $60(24.9)$ & $25(24.0)$ & $35(25.6)$ & \\
\hline $\mathrm{d} 4 \mathrm{~T} / \mathrm{ddl}$ & $17(7.1)$ & $4(3.9)$ & $13(9.5)$ & \\
\hline AZT/ddl & $14(5.8)$ & $3(2.9)$ & $11(8.0)$ & \\
\hline $\mathrm{ddl} / 3 \mathrm{TC}$ & $8(3.3)$ & $1(1.0)$ & $7(5.1)$ & \\
\hline Multi-NRTI resistance ${ }^{2}$ & & & & $<0.001$ \\
\hline No TAMs & $45 / 203(22.2)$ & $38 / 89(42.7)$ & $7 / 114(6.1)$ & \\
\hline Low grade multi-NRTI & 78/203 (38.4) & 40/89 (44.9) & 38/114 (33.3) & \\
\hline High grade multi-NRTI & 80/203 (39.4) & $11 / 89(12.4)$ & 69/114 (60.5) & \\
\hline
\end{tabular}

Data are presented in median (IQR) or number (\%).

${ }^{1}$ Single boosted PI regimens include ritonavir-boosted Protease inhibitor plus 2 nucleoside reverse transcriptase. Double-boosted PI regimens included 2 protease inhibitors plus ritonavir with/without NRTI/NNRTI.

${ }^{2}$ Definition of multi NRTI resistance: high grade is defined as $\geq 4$ thymidine analog associated mutations (TAMs) or the presence of Q151M or 69 Insertion. Low grade multi NRTI resistance is defined as 1-3 TAMs and without Q151M and 69insertion.

vomiting $(\mathrm{n}=2)$, intolerance $(\mathrm{n}=1)$, blurred vision $(\mathrm{n}=1)$, hypertriglyceridemia $(\mathrm{n}=1) ; 5$ events for full dose RTV (31\% of children who used full dose of RTV) due to intolerance $(n=3)$, hyperbilirubinemia $(n=1)$ and hypertriglyceride $(n=1)$; 4 events for NFV ( $10 \%$ of children who used NFV) due to intolerance $(n=2)$, rash $(\mathrm{n}=1)$ and diarrhea $(\mathrm{n}=1)$; 1 event for SQV (4\% of children who used SQV) due to intolerance and 1 event for $\mathrm{LPV} / \mathrm{r}(0.8 \%$ of children who used LPV/r) due to dyslipidemia. Proportion of children with elevated low density lipoprotein was significantly more in the double-boosted compared to single-boosted PI groups (Table 2). 
Table 2 Outcomes after $\mathbf{4 8}$ weeks of boosted protease inhibitor regimens

\begin{tabular}{|c|c|c|c|c|}
\hline \multirow[t]{2}{*}{ Outcomes } & \multicolumn{4}{|l|}{ Week 48} \\
\hline & Total & Single -boosted PI & Double-boosted PI & P-value \\
\hline Median (IQR) CD4\% & $20(15-26)(n=200)$ & $24(18-30)(n=83)$ & $17(14-22)(n=117)$ & $<0.001$ \\
\hline Median(IQR) CD4\% gain & $10(1-7)(n=199)$ & $7(2-12)(n=83)$ & $10(6-15)(n=116)$ & 0.002 \\
\hline$\%$ with HIV RNA < 400 copies/ml & $81.3(n=144)$ & $83.1(n=65)$ & $79.8(n=79)$ & 0.610 \\
\hline$\%$ with HIV RNA < 50 copies/ml & $63.0(n=138)$ & $76.6(n=64)$ & $51.4(n=74)$ & 0.002 \\
\hline$\%$ with Cholesterol > 200 mg/dl & $42.5(n=162)$ & $34.3(n=70)$ & $48.9(n=92)$ & 0.062 \\
\hline$\%$ with Triglyceride > 150 mg/dl & $62.4(n=157)$ & $61.8(n=68)$ & $62.9(n=89)$ & 0.882 \\
\hline$\%$ with Low density lipoprotein > 130 mg/dl & $31.8(n=88)$ & $16.2(n=37)$ & $43.1(n=51)$ & 0.007 \\
\hline
\end{tabular}

\section{Cumulative probability of virologic treatment failure}

The cumulative probability of virologic treatment failure was analyzed by including data from 199 patients who had HIV RNA results at least once after 6 months of boosted PI regimens. The Kaplan Meier estimate is shown in Figure 1. The cumulative probabilities of having HIV RNA > 400 copies $/ \mathrm{ml}$ at 24 months of treatment were 0.28 (95\% CI 0.19-0.40) for children who received single-boosted PI and 0. 27 (95\% CI 0.18-0.39) for children on double-boosted PI, $\mathrm{p}=0.813$ by log rank test.

\section{Predictors for virologic suppression at week 48 after boosted PI regimen}

The predictors for virologic suppression at week 48 were explored by multivariate logistic regression analysis as shown in Table 3. Male, young age and high weight for age z-score were significantly associated with virologic suppression. Male had a 2.9 times odds of achieving viral suppression compared to female. Chances for viral suppression were more likely in children younger than 9 years (3.6 times odds) and those with weight for age $\mathrm{z}$ score $>-1.7$ (4.2 times odds). There was a trend for a better virologic suppression rate among children who switched to boosted PI regimen when their CD4 count was $>100 \mathrm{cell} / \mathrm{mm}^{3}$ or plasma HIV RNA levels $<4 \log _{10}$ copies/ml but these did not reach statistical significance $(\mathrm{p}>0.05)$.

\section{Discussion}

Our study provides information on outcomes of secondline ART in NNRTI-failing children as part of a large multicenter observational study in a RLS. This study showed good virologic efficacy of second-line boosted PI regimens with $81 \%$ achieving $\mathrm{HIV}$ RNA $<400$ copies $/ \mathrm{ml}$ at 48 weeks of treatment. Children with more advanced HIV disease were preferentially treated with doubleboosted PI versus single-boosted PI regimens. Both regimen types performed equally well in suppressing HIV

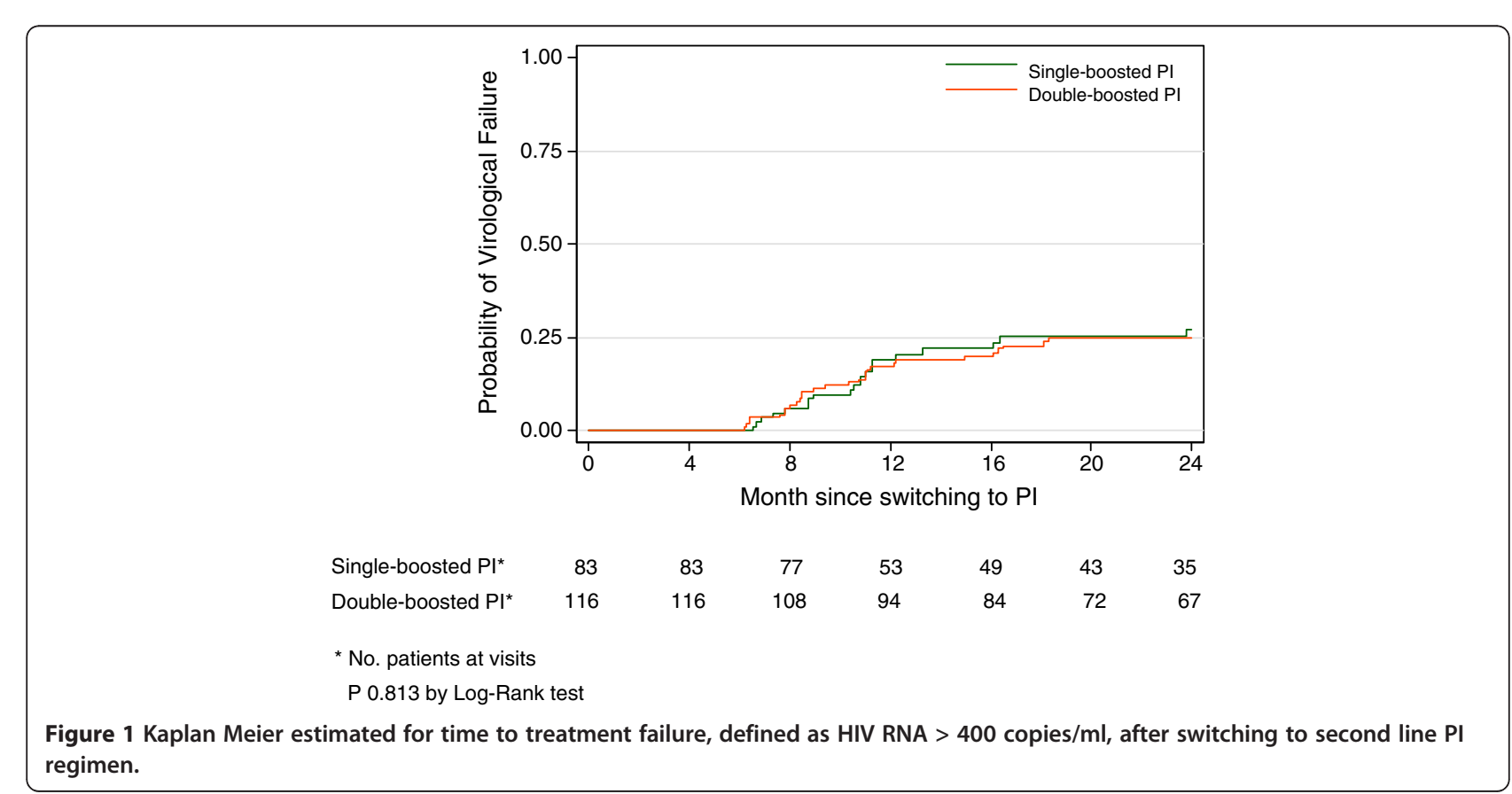


Table 3 The predictors for virological suppression defined as HIV viral load $<400$ copies/ml at week 48 of boosted protease inhibitor-based antiretroviral regimens

\begin{tabular}{|c|c|c|c|c|c|}
\hline & \multirow{2}{*}{$\begin{array}{l}\text { Proportion of children with } \\
\text { viral suppression } \mathrm{n}(\%)\end{array}$} & \multicolumn{2}{|l|}{ Univariate analysis } & \multicolumn{2}{|l|}{ Multivariate analysis } \\
\hline & & Odd Ratios $(95 \% \mathrm{Cl})$ & P-value & Odd Ratios $(95 \% \mathrm{Cl})$ & P-value \\
\hline \multicolumn{6}{|l|}{ ARV regimens } \\
\hline Single-boosted PI & $54 / 65(83.1 \%)$ & 1 & & 1 & \\
\hline Double-boosted PI & $63 / 79(79.8 \%)$ & $0.80(0.34-1.88)$ & 0.611 & $0.82(0.27-2.42)$ & 0.712 \\
\hline \multicolumn{6}{|l|}{ Gender } \\
\hline Male & 62/69 (89.9\%) & $3.22(1.27-8.20)$ & 0.014 & $2.85(1.06-7.66)$ & 0.018 \\
\hline Female & 55/75 (73.3\%) & 1 & & 1 & \\
\hline \multicolumn{6}{|l|}{ Age, years } \\
\hline$<9.1$ & 67/76 (88.2\%) & $2.68(1.12-6.46)$ & 0.028 & $3.60(1.25-10.36)$ & 0.038 \\
\hline$\geq 9.1$ & 50/68 (73.5\%) & 1 & & 1 & \\
\hline \multicolumn{6}{|l|}{ Weight for age Z-score } \\
\hline$<-1.7$ & 42/59 (71.2\%) & 1 & & 1 & \\
\hline$\geq=1.7$ & 64/72 (88.9\%) & $3.24(1.28-8.18)$ & 0.013 & $2.42(0.85-6.84)$ & 0.097 \\
\hline \multicolumn{6}{|c|}{ CD4 at time of switch to second line regimen } \\
\hline$<100 \mathrm{cell} / \mathrm{mm} 3$ & $30 / 42(71.4 \%)$ & 1 & & 1 & \\
\hline$\geq 100 \mathrm{cell} / \mathrm{mm} 3$ & $84 / 99$ (84.9\%) & $2.24(0.94-5.33)$ & 0.068 & $2.12(0.71-6.36)$ & 0.178 \\
\hline \multicolumn{6}{|c|}{ Plasma HIV RNA at time of switch } \\
\hline$<4 \log _{10}$ copies $/ \mathrm{ml}$ & 25/29 (86.2\%) & $1.53(0.48-4.86)$ & & - & - \\
\hline$\geq 4 \log _{10}$ copies $/ \mathrm{ml}$ & 25/111 (80.4\%) & 1 & 0.474 & & \\
\hline \multicolumn{6}{|l|}{ Mono/dual NRTI exposure } \\
\hline Yes & $41 / 50(82.0 \%)$ & $1.08(0.45-2.62)$ & 0.867 & & \\
\hline No & 76/94 (80.9\%) & 1 & & & \\
\hline \multicolumn{6}{|l|}{ Duration on NNRTI-based } \\
\hline$<2.2$ year & 69/84(82.1\%) & $1.15(0.50-2.67)$ & 0.745 & & \\
\hline$\leq 2.2$ year & $48 / 60(80.0 \%)$ & 1 & & & \\
\hline \multicolumn{6}{|l|}{ NNRTI -based regimen } \\
\hline NVP-based & $81 / 96(84.4 \%)$ & $1.80(0.77-4.23)$ & 0.178 & $1.24(0.43-3.55)$ & 0.691 \\
\hline EFV-based & $36 / 48(75.0 \%)$ & 1 & & 1 & \\
\hline Multi-NRTI resistance & & & 0.809 & - & - \\
\hline No TAMs & 25/32 (78.1\%) & 1 & & & \\
\hline Low grade multi-NRTI & $35 / 44$ (79.6\%) & $1.09(0.36-3.31)$ & & & \\
\hline High grade multi-NRTI & 36/43 (83.7\%) & $1.44(0.45-4.62)$ & & & \\
\hline \multicolumn{6}{|l|}{$\mathrm{LPV} / \mathrm{r}$ in the regimen } \\
\hline Yes & $60 / 71$ (84.5\%) & $1.53(0.66-3.58)$ & 0.325 & & \\
\hline No & $57 / 73(78.1 \%)$ & 1 & & & \\
\hline
\end{tabular}

below 400 copies/ml but further suppression to less than 50 copies/ml was significantly less with double-boosted PI.

This study supports recycling of 2NRTIs in combination with a potent boosted PI as second-line therapy in children. The $2010 \mathrm{WHO}$ guideline recommend replacing $\mathrm{AZT} / 3 \mathrm{TC}$ or $\mathrm{d} 4 \mathrm{~T} / 3 \mathrm{TC}$ in the first line regimen with abacavir $(\mathrm{ABC}) / 3 \mathrm{TC}$ or $\mathrm{ABC} / \mathrm{ddI}$ [17]; however, that practice is rare in Thailand due to the high cost of $\mathrm{ABC}$ rendering it unavailable in the Thai national ARV program. Therefore, recycling of inactive or partially active 2NRTIs such as AZT/3TC, AZT/ddI or ddI/3TC was common in this study. At the time of the study, tenofovir (TDF) was not yet approved for adolescents nor was it available in Thailand. Similarly, LPV/r is the 
PI of choice in the WHO guideline but it was in limited supply in Thailand before 2005, resulting in the use of a more toxic drug, IDV, in half of our children.

The virologic response to second-line single boosted PI regimen in our study compares favorably to reports from other countries [15-17]. In a French cohort, 92\% on LPV/r second line regimens had HIV RNA $<400$ copies/ml despite having lower CD4 (14.8\%) and higher HIV RNA (4.8 $\log _{10}$ copies/ml) than our children. The Spanish multicenter retrospective observational study showed $71.5 \%$ of PI-experienced children on LPV/rbased having HIV RNA $<400$ copies/ml [10]. A long term cohort of children treated with $\mathrm{LPV} / \mathrm{r}$-based regimen showed that $81 \%$ remained on therapy after more than 4 years; $75 \%$ of those children had HIV RNA $<400$ copies/ml at their last visit. [18] In another pediatric study, patients tended to have better virologic outcome if they were on LPV/r as supposed to unboosted PI, nelfinavir [19]. We also saw a trend towards better virologic outcome among children who received LPV/r, but this did not reach statistical significance. In Malawi, $10 \%$ of adults died after initiating second-line regimen and 85\% of the survivors had viral suppression at 12 months [20].

Double-boosted PI is an acceptable alternative secondline therapy option in Thailand for children with late treatment failure who have few or no fully active drugs aside from PIs [15]. The pediatric HIV-NAT 017 study which treated children with second-line SQV and LPV/r reported a viral suppression rate similar to that observed in our study with $64 \%$ having HIV RNA $<50$ copies $/ \mathrm{ml}$ at week 48 [21]. A non-randomized study in Thai adults showed that the double-boosted PI regimen was not as potent in suppressing HIV RNA to below 50 copies $/ \mathrm{ml}$ when compared to the single-boosted PI regimen among patients with low grade multi- NRTI resistance mutations [22]. Of note is the inability of that study to compare the two regimen types among patients with high grade multi-NRTI resistance mutations. Our study showed that double-boosted PI had a higher rate of low level viremia, HIV RNA between 50 and 400 copies/ml, compared to single-boosted PI. This could possibly be confounded by indication that children with more advanced disease received double-boosted PI regimen or due to inferior potency of a PI mono-class regimen. A recent randomized study of second-line PIs in Thai adults showed mono LPV/r treatment to result in significantly more low level viremia than a 3-drug regimen with TDF, 3TC and LPV/r [23]. Another second-line study in Thai adults who received a single active drug, $\mathrm{LPV} / \mathrm{r}$, together with 3TC to reduce viral fitness also showed that only $67 \%$ achieved HIV RNA $<50$ copies $/ \mathrm{ml}$ with $16 \%$ having low viremia between $50-400$ copies $/ \mathrm{ml}$ [24]. The recent review literature [25] and meta-analysis [26] on protease inhibitor monotherapy also showed a slightly inferior virological efficacy of protease inhibitor monotherapy than that of protease inhibitor plus nucleosides. However, failure of protease inhibitor monotherapy does not imply losing therapeutic options, usually reintroduction of nucleosides can lead to virologic suppression. [25,26] The higher rates of dyslipidemia with double-boosted PI and the higher pill burden further limits its use particularly if newer and more potent ARVs with favorable lipid profile e.g. atazanavir, darunavir, and raltegravir becomes available to children in RLS.

There is a lack of evidence to inform the optimum time to switch to second line regimens in RLS. The WHO guideline, relying heavily on a public health approach to care, recommends switching to second-line regimen when CD4 is $<100$ cells $/ \mathrm{mm}^{3}$ or HIV RNA is $>$ 5,000 copies $/ \mathrm{ml}$ [27]. We demonstrated a trend towards better virologic suppression following second-line therapy in children who switched when their CD4 was $>100$ cell $/ \mathrm{mm}^{3}$ or HIV RNA was $<10,000$ copies $/ \mathrm{ml}$. Delayed in switching to second-line therapy may lead to resistance mutations accumulation which would be problematic in RLS where ARV options are limited. The PENPACT-1 study showed that children failing first-line NNRTI who were randomized to a delayed switch at HIV RNA > 30,000 copies/ml had more TAMs compared to those who switched at HIV RNA $>1,000$ copies $/ \mathrm{ml}$ [28] This however did not affect the overall virologic outcome after second-line therapy in that study likely due to several reasons: children who failed had low rates of NRTI resistance of $1-5 \%$, they had no CD4 failure, and they had access to routine HIV RNA monitoring, genotyping and good ARV options. The situation in RLS may be quite different as switching occurs later with immunologic or clinical failure coupled with the lack of HIV RNA and genotyping monitoring, and limited ARV options.

This study has several limitations. First is incomplete data, which is an inherent limitation of a retrospective study design. The information of adherence to treatment is not captured. Second, the lack of randomization of single- versus double-boosted PI regimens hinders the ability to directly compare the outcome between these two regimens. Third, the duration of NNRTI-based first line regimen in our study was relatively short, around 2 years. Furthermore, we have access to laboratory monitoring including CD4, plasma HIV RNA and genotypic resistance testing to guide the design of the new regimens which may limit the applicability of our results to other resource-limtted settings where treatment failure is detected later and genotyping is unavailable. However, the constraint in available ARV choices in our population prohibited us to fully utilize genotyping information. Therefore, we believe that our data represent typical clinical care settings in most resource-limited countries. 
In conclusion, this study provides security to clinicians in RLS to give children second-line treatment despite drug choice and laboratory monitoring constraints. Although we saw a good success rate in our study, onefifth did not achieve viral suppression. Such children in RLS currently have no treatment option beyond secondline regimens. This emphasizes the urgent need to derive strategies to study, plan and procure new drugs and drug classes for children with treatment failure worldwide.

\section{The HIV-NAT 086 study team}

The HIV Netherlands Australia Thailand Research Collaboration (HIV-NAT), The Thai Red Cross AIDS Research Centre, Bangkok: T. Bunupuradah, C. Phasomsap, P. Kaew-on.

Institut de Recherche pour le Développement (IRD), UMI 174, Program for HIV Prevention and Treatment, Department of Medical Technology, Faculty of Associated Medical Sciences, Chiang Mai University, Chiang Mai, Thailand, The Global Fund to Fight AIDS, Malaria and Tuberculosis supported drug and laboratory monitoring for some children: S Kanjanavanit, Nakornping Hospital, Somdej Pranangchao Sirikit Hospital, Chonburi, Somdej Pranangchao Sirikit Hospital, Chonburi: T Hinjiranandana, Bhumibol Adulyadej Hospital, Bangkok: P Layangool, Somdej Prapinklao Hospital, Bangkok: N Kamonpakorn, Mae Chan Hospital, Chiang Rai: S Buranabanjasatean, Prapokklao Provincial Hospital, Chantaburi: C Ngampiyaskul.

Queen Sirikit National Institute of Child Health, Bangkok: T. Chotpitayasunondh, S. Chanpradub, P. Leawsrisuk. Department of Pediatrics, Faculty of Medicine, Siriraj Hospital, Mahidol University: S. Chearskul, N. Vanprapar, W. Phongsamart, K. Lapphra, P. Chearskul, O. Wittawatmongkol, W. Prasitsuebsai, K. Intalapaporn, N. Kongstan, N. Pannin, A. Maleesatharn, B. Khumcha.

Research Institute for Health Sciences (RIHES), Chiang Mai University, Chiang Mai: L. Aurpibul, N. Wongnum, R. Nadsasarn.

Department of Pediatrics, Faculty of Medicine, Khon Kaen University: P. Lumbiganon, P. Tharnprisan, T. Udompanich. Petchburi Hospital, Petchburi: M. Yentang.

Chiang Rai Regional Hospital, Chiang Rai: A. Khonponoi, N. Maneerat, S. Denjunta, S. Watanaporn, C. Yodsuwan, W. Srisuk, S. Somsri, K. Surapanichadul

\section{Competing interests}

All authors declare no conflict of interest and that member of their immediate families do not have a financial interest in or arrangement with any commercial organization that may have a direct interest in the subject matter of this article.

\section{Authors's contribution}

$T P, G J, K C$, and JA designed the study, collected data, wrote the first draft, reviewed manuscript before submission. PS, TS,WP,VS, PK,WP,RH, NW collected data, reviewed and commented of manuscript before submission.
US analyzed, reviewed and commented draft of manuscript before submission. All authors have read and approved the final manuscript.

\section{Acknowledgements}

The study was funded by the Commission on Higher Education, Ministry of Education in Thailand. The data were collected from the Pediatric PHPT cohort study $(\mathrm{N}=74)$, Queen Sirikit National Institute of Child Health, Bangkok ( $N=53)$, HIVNAT, Thai Red Cross AIDS Research Center, Bangkok ( $N=44)$, Siriraj Hospital, Mahidol University, Bangkok $(N=28)$, Chiang Mai University hospital, Chiang Mai $(\mathrm{N}=18)$, Khon Kaen University, Khon Kaen $(\mathrm{N}=16)$, Petchburi Provincial Hospital, Petchburi $(\mathrm{N}=4)$ and Chiang Rai Regional Hospital, Chiang Rai $(\mathrm{N}=4)$. We would like to thank Frank Benjamin Steven, MD for his help in preparing the English manuscript.

\section{Author details}

${ }^{1}$ The HIV Netherlands Australia Thailand Research Collaboration (HIV-NAT), Bangkok, Thailand. 'Department of Pediatrics, Faculty of Medicine, Chulalongkorn University, Bangkok, Thailand. ${ }^{3}$ Institut de Recherche pour le Développement (IRD), UMI 174, Program for HIV Prevention and Treatment, Department of Medical Technology, Faculty of Associated Medical Sciences, Chiang Mai University, Chiang Mai, Thailand. ${ }^{4}$ Queen Sirikit National Institute of Child Health, Bangkok, Thailand. ${ }^{5}$ Department of Pediatrics, Faculty of Medicine Siriraj Hospital, Mahidol University, Bangkok, Thailand. ${ }^{6}$ Research Institute for Health Sciences, Chiang Mai University, Chiang Mai, Thailand. ${ }^{7}$ Department of Pediatrics, Faculty of Medicine, Khon Kaen University, Khon Kaen, Thailand. ${ }^{8}$ Petchburi Hospital, Petchburi, Thailand. ${ }^{9}$ Chiang Rai Regional Hospital, Chiang Rai, Thailand. ${ }^{10}$ South East Asia Research Collaboration with Hawaii, Hawaii, Thailand. " Department of Medicine, Faculty of Medicine, Chulalongkorn University, Bangkok, Thailand.

Received: 14 December 2011 Accepted: 9 June 2012

Published: 18 June 2012

\section{References}

1. Ciaranello AL, Chang Y, Margulis AV, Bernstein A, Bassett IV, Losina E, et al: Effectiveness of pediatric antiretroviral therapy in resource-limited settings: a systematic review and meta-analysis. Clin Infect Dis 2009 Dec 15, 49(12):1915-27.

2. Kariminia A, Chokephaibulkit K, Pang J, Lumbiganon P, Hansudewechakul R, Amin J, et al: Cohort profile: the TREAT Asia pediatric HIV observational database. Int J Epidemiol 2011 Feb, 40(1):15-24.

3. Puthanakit T, Oberdorfer A, Akarathum N, Kanjanavanit S, Wannarit P, Sirisanthana T, et al: Efficacy of highly active antiretroviral therapy in HIVinfected children participating in Thailand's National Access to Antiretroviral Program. Clin Infect Dis 2005 Jul 1, 41(1):100-7.

4. Kamya MR, Mayanja-Kizza H, Kambugu A, Bakeera-Kitaka S, Semitala F, Mwebaze-Songa $P$, et al: Predictors of long-term viral failure among ugandan children and adults treated with antiretroviral therapy. Journal of acquired immune deficiency syndromes 2007, 46(2):187-93.

5. Janssens B, Raleigh B, Soeung S, Akao K, Te V, Gupta J, et al: Effectiveness of highly active antiretroviral therapy in HIV-positive children: evaluation at 12 months in a routine program in Cambodia. Pediatrics 2007 Nov, 120 (5):e1134-40.

6. Jaspan HB, Berrisford AE, Boulle AM: Two-year outcomes of children on non-nucleoside reverse transcriptase inhibitor and protease inhibitor regimens in a South African pediatric antiretroviral program. Pediatr Infect Dis J 2008, 27(11):993-8.

7. Prasitsuebsai W, Bowen AC, Pang J, Hesp C, Kariminia A, Sohn AH: Pediatric HIV clinical care resources and management practices in Asia: a regional survey of the TREAT Asia pediatric network. AIDS Patient Care STDS 2010, 24(2):127-31.

8. PENTA 2009 guidelines for the use of antiretroviral therapy in paediatric HIV-1 infection. Available at: $\leq$ http://www.pentatrials.org/guide09.pdf $\geq$ (Accessed: July 31, 2009)

9. Working Group on Antiretroviral Therapy and Medical Managment of HIV-infected children: Guidelines for the Use of Antiretroviral Agents in Pediatric HIV Infection, February 23, 2009. US Department of Health and Human Services: Access; 2009. http://AIDSinfo.nih.gov/.

10. Resino S, Bellon JM, Ramos JT, Navarro ML, Martin-Fontelos P, Cabrero E, et al: Salvage lopinavir-ritonavir therapy in human immunodeficiency virus-infected children. Pediatr Infect Dis J 2004, 23(10):923-30. 
11. Sungkanuparph S, Manosuthi W, Kiertiburanakul S, Piyavong B, Chumpathat N, Chantratita W: Options for a second-line antiretroviral regimen for HIV type 1-infected patients whose initial regimen of a fixed-dose combination of stavudine, lamivudine, and nevirapine fails. Clin Infect Dis 2007 Feb 1, 44(3):447-52.

12. Jittamala $P$, Puthanakit $T$, Chaiinseeard $S$, Sirisanthana V: Predictors of virologic failure and genotypic resistance mutation patterns in thai children receiving non-nucleoside reverse transcriptase inhibitor-based antiretroviral therapy. Pediatr Infect Dis J 2009, 28(9):826-30.

13. Puthanakit T, Jourdain G, Hongsiriwon S, Suntarattiwong P, Chokephaibulkit $K$, Sirisanthana $V$, et al: HIV-1 drug resistance mutations in children after failure of first-line nonnucleoside reverse transcriptase inhibitor-based antiretroviral therapy. HIV medicine 2010, 11(9):565-72.

14. Bunupuradah T, van der Lugt J, Kosalaraksa P, Engchanil C, Boonrak P, Puthanakit T, et al: Safety and efficacy of a double-boosted protease inhibitor combination, saquinavir and lopinavir/ritonavir, in pretreated children at 96 weeks. Antivir Ther 2009, 14(2):241-8.

15. Thai Ministry of Public Health: Thai National guideline for managment of HIV-infected children and adults; 2004. ISBN 974-297-2982.

16. Sirivichayakul S, Ruxrungtham K, Ungsedhapand C, Techasathit W, Ubolyam S, Chuenyam T, et al: Nucleoside analogue mutations and Q151M in HIV-1 subtype A/E infection treated with nucleoside reverse transcriptase inhibitors. AIDS (London, England) 2003, 17(13):1889-96.

17. World Health Organization: Antiretroviral therapy for HIV infection in infants and children: towards universal access.: Recommendations for a public health approach; 2010.

18. Kline MW, Rugina S, llie M, Matusa RF, Schweitzer AM, Calles NR, et al: Long-term follow-up of 414 HIV-infected Romanian children and adolescents receiving lopinavir/ritonavir-containing highly active antiretroviral therapy. Pediatrics 2007 May, 119(5):e1116-20.

19. De Luca M, Miccinesi G, Chiappini E, Zappa M, Galli L, De Martino M: Different kinetics of immunologic recovery using nelfinavir or lopinavir/ ritonavir-based regimens in children with perinatal HIV-1 infection. Int $J$ Immunopathol Pharmacol 2005, 18(4):729-35.

20. Hosseinipour MC, Kumwenda JJ, Weigel R, Brown LB, Mzinganjira D, Mhango B, et al: Second-line treatment in the Malawi antiretroviral programme: high early mortality, but good outcomes in survivors, despite extensive drug resistance at baseline. HIV medicine 2010, 11(8):510-8.

21. Kosalaraksa $P$, Bunupuradah $T$, Engchanil C, Boonrak P, Intasan J, Lumbiganon $P$, et al: Double boosted protease inhibitors, saquinavir, and lopinavir/ritonavir, in nucleoside pretreated children at 48 weeks. Pediatr Infect Dis J 2008, 27(7):623-8.

22. Chetchotisakd P, Anunnatsiri S, Mootsikapun P, Kiertiburanakul S, Anekthananon T, Bowonwatanuwong C, et al: Efficacy and tolerability of a double boosted protease inhibitor (lopinavir + saquinavir/ritonavir) regimen in HIV-infected patients who failed treatment with nonnucleoside reverse transcriptase inhibitors. HIV medicine 2007, 8(8):529-35.

23. Bunupuradah T, Chetchotisakd P, Ananworanich J, Munsakul W, Jirajariyavej $S$, Kantipong $P$, et al: A randomized comparison of second-line lopinavir/ ritonavir monotherapy vs. tenofovir/lamivudine/lopinavir/ritonavir in patients failing NNRTI-regimens: the HIV STAR study. Antivir Ther 2012, Jul 2 [Epub ahead of print].

24. Manosuthi W, Kiertiburanakul S, Amornnimit W, Prasithsirikul W, Thongyen S, Nilkamhang S, et al: Treatment outcomes and plasma level of ritonavirboosted lopinavir monotherapy among HIV-infected patients who had NRTI and NNRTI failure. AIDS Res Ther 2009, 6:30.

25. Perez-Valero I, Arribas JR: Protease inhibitor monotherapy. Curr Opin Infect Dis, 24(1):7-11.

26. Mathis S, Khanlari B, Pulido F, Schechter M, Negredo E, Nelson M, et al: Effectiveness of protease inhibitor monotherapy versus combination antiretroviral maintenance therapy: a meta-analysis. PLoS One 2011, 6(7): e22003.

27. Antiretroviral therapy for HIV infection in infants and children towards universal access Recommendation for public health approach: 2010 revision; Available at: $\leq$ http://www.who.int/hiv/topics/paediatric/en/ index.html $\geq$ (Access September 2, 2010).
28. Babiker A, Castro nee Green H, Compagnucci A, Fiscus S, Giaquinto C, Gibb $\mathrm{DM}$, et al: First-line antiretroviral therapy with a protease inhibitor versus non-nucleoside reverse transcriptase inhibitor and switch at higher versus low viral load in HIV-infected children: an open-label, randomised phase 2/3 trial. Lancet Infect Dis 2011, (4):273-83.

doi:10.1186/1742-6405-9-20

Cite this article as: Puthanakit et al:: High virologic response rate after second-line boosted protease inhibitor-based antiretroviral therapy regimens in children from a resource limited setting. AIDS Research and Therapy 2012 9:20.

\section{Submit your next manuscript to BioMed Central and take full advantage of:}

- Convenient online submission

- Thorough peer review

- No space constraints or color figure charges

- Immediate publication on acceptance

- Inclusion in PubMed, CAS, Scopus and Google Scholar

- Research which is freely available for redistribution 